\title{
Insulin Pump Treatment in Adults with Type 1 Diabetes in the Capital Region of Denmark: Design and Cohort Characteristics of the Steno Tech Survey
}

\author{
Karen Rytter (1) - Kristoffer P. Madsen (D) - Henrik U. Andersen (1) - \\ Bryan Cleal (1) · Eva Hommel (1) · Mette A. Nexø (1) · \\ Ulrik Pedersen-Bjergaard (D) - Timothy Skinner (1) · Ingrid Willaing (1) · \\ Kirsten Nørgaard (i) $\cdot$ Signe Schmidt (1)
}

Received: October 3, 2021 / Accepted: November 3, 2021 / Published online: November 22, 2021

(c) The Author(s) 2021

\begin{abstract}
Introduction: Insulin pump therapy can improve quality of life and glycaemic outcomes for many people with type 1 diabetes (T1D). The multidimensional Steno Tech Survey study aims
\end{abstract}

Karen Rytter and Kristoffer P. Madsen have contributed equally to this work and are considered co-first authors.

Supplementary Information The online version contains supplementary material available at https:// doi.org/10.1007/s13300-021-01181-0.

K. Rytter · H. U. Andersen - E. Hommel .

K. Nørgaard · S. Schmidt $(\bowtie)$

Clinical Research, Copenhagen University Hospital

- Steno Diabetes Center Copenhagen, Herlev,

Denmark

e-mail: signe.schmidt@regionh.dk

H. U. Andersen

e-mail: henrik.ullits.andersen@regionh.dk

E. Hommel

e-mail: eva.hommel@regionh.dk

K. Rytter · U. Pedersen-Bjergaard · K. Nørgaard Department of Clinical Medicine, Faculty of Health and Medical Sciences, University of Copenhagen, Copenhagen, Denmark

K. Rytter

e-mail: karen.rytter@regionh.dk

K. Nørgaard

e-mail: kirsten.noergaard@regionh.dk to investigate why some insulin pump users do not achieve treatment goals. In this article, we present the study design and analyse differences in population characteristics between responders and non-responders.

Methods: In June 2020, all 1591 insulin pump users ( $\geq 18$ years) in the Capital Region of Denmark were invited to participate in an online questionnaire that evaluated several dimensions of insulin pump self-management and psychosocial health. Demographic,

K. P. Madsen · B. Cleal · M. A. Nexø · I. Willaing Health Promotion Research, Copenhagen University Hospital - Steno Diabetes Center Copenhagen, Herlev, Denmark

B. Cleal

e-mail: bryan.richard.cleal@regionh.dk

M. A. Nexø

e-mail: mette.andersen.nexoe@regionh.dk

K. P. Madsen

Danish Centre for Health Economics, University of Southern Denmark, Odense, Denmark

e-mail: kristoffer.panduro.madsen@regionh.dk

U. Pedersen-Bjergaard

Department of Endocrinology and Nephrology, Nordsjællands Hospital, Hillerød, Denmark e-mail: ulrik.pedersen-bjergaard@regionh.dk

T. Skinner

Department of Psychology, University of

Copenhagen, Copenhagen, Denmark

e-mail: t.skinner@latrobe.edu.au 
socioeconomic and clinical characteristics, including age, sex and HbA1c, of the cohort were identified via national registries. Predictors of questionnaire response/non-response were explored with logistic regression analysis.

Results: In the full study population, 58\% were female, median age was 42 years and median HbA1c was $58 \mathrm{mmol} / \mathrm{mol}$ (7.5\%); 30\% had $\mathrm{HbA} 1 \mathrm{c}<53 \mathrm{mmol} / \mathrm{mol}$ (7.0\%). In total, 770 individuals $(48 \%)$ responded to the questionnaire. Logistic regression analysis showed that $50+$ years of age (odds ratio $[\mathrm{OR}]=2.3,95 \%$ confidence interval [CI]: 1.4-3.8), female sex $(\mathrm{OR}=1.3$, CI: $1.02-1.6)$, being married $(\mathrm{OR}=$ 1.8, CI: 1.3-2.4) and having long higher education $(\mathrm{OR}=1.6, \mathrm{CI}$ : $1.004-2.5)$ were significantly associated with a higher likelihood of responding to the survey; the opposite was found for $\mathrm{HbA} 1 \mathrm{c}$ from 64 to $<75 \mathrm{mmol}$ $(8.0-9.0 \%) \quad(\mathrm{OR}=0.6, \quad \mathrm{CI}: \quad 0.4-0.8) \quad$ and $\mathrm{HbA} 1 \mathrm{c} \geq 75 \mathrm{mmol} / \mathrm{mol}(\geq 9.0 \%)(\mathrm{OR}=0.2, \mathrm{CI}$ : 0.1-0.3).

Conclusions: The established Steno Tech cohort enables future analysis of a range of psychosocial and behavioural aspects of insulin pump self-management. Interpretation and generalization of findings should consider observed differences between responders and non-responders.

Keywords: HbA1c; Insulin pump; National registries; Response/non-response; Survey; Type 1 diabetes

\section{Key Summary Points}

Why carry out this study?

-Insulin pump therapy can improve quality of life and glycaemic control for many people with type 1 diabetes; however, heterogeneity in outcomes persist, and little is known about reasons for this.

-Analysis of the Steno Tech data may inform interventions aimed at improving outcomes of insulin pump treatment taking differences between responders and non-responders into account.

\section{What was learned from the study?}

-The Steno Tech Survey cohort of insulin pump users with type 1 diabetes was established using real-world clinical outcomes data from national registries and an elaborate questionnaire-based survey to assess insulin pump practices and psychosocial health.

-Questionnaire responders were older and had lower Hba1c compared with nonresponders, but did not differ regarding annual income, diabetes duration, longterm diabetes complications and other comorbidities.

-This study may serve as an example of how to assess non-response bias and posits that future studies should focus especially on reaching young people and those facing challenges in meeting glycaemic targets.

\footnotetext{
I. Willaing

Department of Public Health, University of

Copenhagen, Copenhagen, Denmark

e-mail: ingrid.willaing.tapager@regionh.dk

S. Schmidt

Department of Clinical Pharmacology, Bispebjerg and Frederiksberg Hospital, Copenhagen, Denmark
}

\section{INTRODUCTION}

Insulin pump therapy has the potential to improve glycaemic outcomes and quality of life for people living with type 1 diabetes (T1D), but not everyone realizes these benefits [1]. An estimated $63 \%$ of adults with T1D in the US and 
$5-15 \%$ of those in Europe use an insulin pump $[2,3]$. Recent observational data from the T1D Exchange Registry show that while not reaching HbA1c target on average, people using insulin pumps achieve better metabolic outcomes compared with people using multiple daily injections (MDI). Additionally, combining insulin pumps with continuous glucose monitoring (CGM) is associated with further improved outcomes [3]. Similar findings have been reported in other observational studies [4-6] and randomized controlled trials [7-10]. It is recognized, however, that reaping the benefits of these technologies requires high levels of engagement from users and health care professionals (HCPs) and that outcomes vary greatly among individuals $[1,11,12]$.

In Denmark, health care is publicly funded, and it is estimated that $19 \%$ of adults with T1D are treated with an insulin pump [13]. Treatment of T1D primarily takes place in outpatient clinics and treatment with insulin pumps only in outpatient clinics specialized in insulin pump management. The National Board of Health states criteria for which individuals can initiate insulin pump treatment, the most important one being not having achieved a HbA1c target of $53 \mathrm{mmol} / \mathrm{mol}$ (7.0\%) [14]. However, large regional differences in insulin pump uptake remain as budgets and policies can overrule professional decisions.

Self-management of T1D, including the use of insulin pumps and CGMs, is a complex social and behavioural process, which ideally involves a comprehensive understanding of glucose control and advanced functions of devices [11]. As such, to ensure safe and effective insulin pump use, it is recommended that people with T1D receive structured and recurring education $[11,12,15]$. However, there is currently no consensus on the best educational practice for a given patient $[15,16]$, and there is a paucity of high-quality comparative studies regarding the effectiveness of insulin pump education [17-19]. Furthermore, psychosocial factors, such as self-efficacy, emotional (diabetes) distress and fear of hypoglycaemia, may also impact the use of technology and treatment outcomes, although the number of quantitative studies analysing the impact of these factors is limited [20, 21]. Qualitative studies exploring how people live with an insulin pump suggest a variety of factors which may affect glucose levels and treatment satisfaction [22, 23]. For example, life conditions, such as close relationships, work and leisure, all play important roles in how the individual acts in everyday life with the insulin pump, and the decision about what target blood glucose the individual is aiming for is situational. As such, integration of devices with everyday life is an ongoing and often challenging negotiation in which diabetes control is balanced against being controlled by diabetes [24]. Further investigation of factors that both influence and are influenced by use of diabetes technology, such as psychosocial factors, is needed to identify targets of intervention in insulin pump treatment.

The Steno Tech Survey is a questionnaire and register-based multidimensional study of adult insulin pump users with T1D in the Capital Region of Denmark. It aims to generate new insights into the state of, and reasons for, variations in outcomes of insulin pump therapy by combining knowledge about diabetes device use and self-management with psychosocial aspects, demographics and socioeconomic status. In this article, we describe the development and execution of the Steno Tech Survey study, report cohort characteristics and analyse these for differences between questionnaire-responders and non-responders to assess representativeness of responders.

\section{METHODS}

\section{Study Design and Population}

The study included data from a cross-sectional, questionnaire-based online survey, data from electronic medical records (EMRs) and data from Danish national registries. Adults ( $\geq 18$ years) with T1D treated with an insulin pump (with or without CGM or intermittently scanned [is]CGM) attending Steno Diabetes Center Copenhagen (SDCC, $n=1423$ ) or Nordsjællands Hospital Hillerød (NOH, $n=168$ ) were invited to participate in the survey $(n=1591)$ (Fig. 1). 


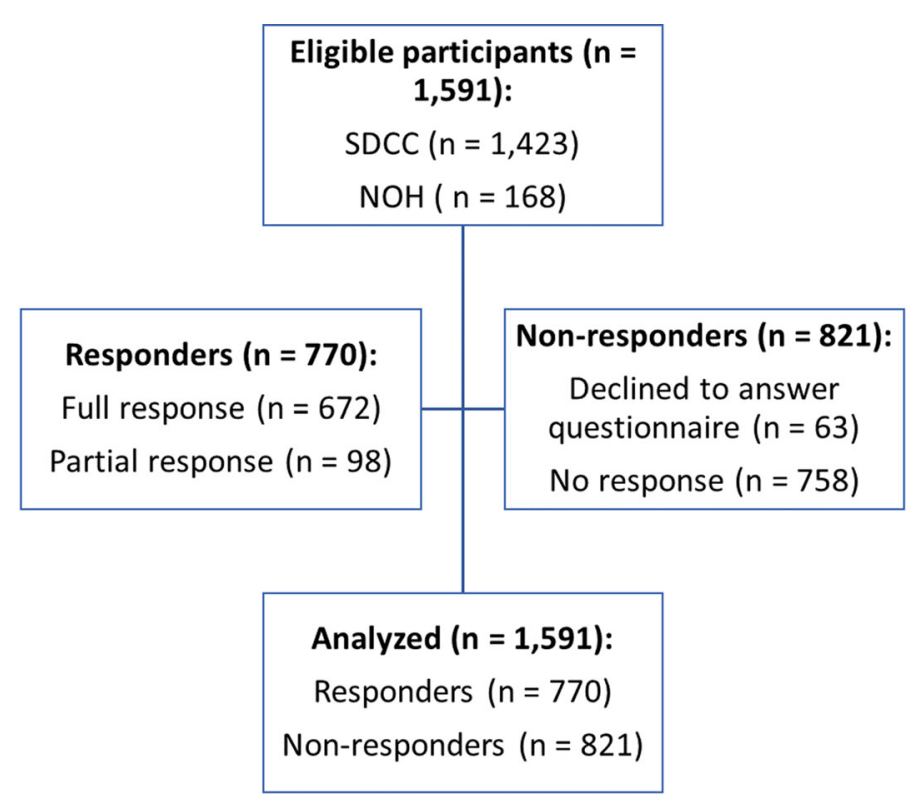

Fig. 1 Flow chart of recruitment procedure. SDCC Steno Diabetes Center Copenhagen, $N O H$ Nordsjællands Hospital Hillerød

\section{Recruitment}

Potential study participants were identified via local registries of insulin pump users at the two hospitals (SDCC and NOH). Eligible study participants were invited to participate via an electronic mailing system (e-Boks), which is the primary way of communication between Danish authorities and citizens, including the health care sector. The invitation thoroughly described the study purpose and content to ensure participant fidelity and contained a link to the online questionnaire. Participants provided digital informed consent before commencing with the questionnaire.

The study was approved by the Danish Data Protection Agency (P-2019-812), exempted from review by the Capital Region of Denmark's Research Ethics Committee (19080899) under the National Danish Research Ethics Committee and conducted in accordance with the Helsinki Declaration of 1964 and its later amendments. The study was also registered on ClinicalTrials.gov (NCT04311164).

\section{Data Collection}

The questionnaire was administered with REDCap, an online data and questionnaire management tool [25]. The questionnaire was open from 15 June to 5 October 2020. The questionnaire was distributed after the first COVID-19 lockdown ended and closed before the second lockdown began in Denmark. Once the questionnaire was closed, responses were merged with data from respondents' EMRs and national Danish registries. Non-responders and partial completers received a reminder 2 weeks after the opening date and again 1 month later.

\section{Demographic and Socioeconomic Data}

Data on demographics and socioeconomic status were obtained from national Danish registries, including the Population Registry and the Civil Registration System [26], the Education Registry [27] and the Income Registry [28]. Variables included age, sex, marital and cohabitation status, education level, employment status and annual personal and family income. 


\section{Clinical Data}

Information on biochemical markers, including HbA1c, cholesterol, triglycerides, urine albumin to creatinine ratio (UACR) and thyrotropin (TSH), was obtained from the Danish National Laboratory Database [29]. The latest available HbA1c value within 1 year of the survey distribution date was used. Because the remaining biochemical outcomes are only routinely measured annually, the latest available value within 2 years of the survey date was used. Data on medical history from the previous five years including acute and chronic diabetes-related complications and comorbidities were obtained from the Danish National Patient Registry [30]. A 5-year Charlson Comorbidity Index score was calculated based on prevalent comorbidities [31]. EMRs of respondents were manually reviewed for information on hypoglycaemia awareness, body mass index (BMI) and blood pressure. Hypoglycaemia awareness was categorized as per Pedersen-Bjergaard et al. [32].

\section{Questionnaire Development}

Questionnaire content was informed by preceding works by the Steno Tech study group, including systematic literature reviews $[19,20,33]$ and an interview study with insulin pump users [24] that explored possibilities and barriers to effective daily insulin pump management. The Steno Tech study group is an interdisciplinary collaboration of researchers and clinicians with extensive experience in type 1 diabetes and insulin pump treatment. The study group includes capacities within endocrinology and diabetes nursing, anthropology, psychology and public health.

The preliminary version of the entire questionnaire was initially pilot tested with seven HCPs experienced in insulin pump therapy but not otherwise involved in the study (four diabetes nurse specialists, one endocrinologist and two dietitians), followed by a pilot test with six people with T1D purposefully sampled regarding age, profession, complication status and insulin pump type. Cognitive interviewing with people with T1D led to minor revisions of content and language-related shortcomings, and comprehensiveness of response categories for constructed items was confirmed [34]. To explore any technical issues in the questionnaire, it was tested in a trial run with 50 randomly selected potential participants, of which 13 responded within 2 weeks. No conceptual or semantical changes were made to the questionnaire based on this trial run; however, some minor structural changes and clarifications in the invitation letter were implemented. Thus, the trial run sample was included as responders in the final sample.

\section{Questionnaire Content}

The final questionnaire consisted of six parts: (1) insulin pump and glucose monitoring device characteristics and utilized functions and device satisfaction measured with the Insulin Device Satisfaction [35] and Glucose Monitoring Satisfaction [36] surveys; (2) insulin pump education needs and preferences; (3) assessment of carbohydrate counting skills; (4) general health behaviour regarding diet, smoking, alcohol intake and physical activity [37]; (5) general and diabetes-specific psychosocial health, including the Type 1 Diabetes Distress Scale [38], the WHO-5 Well-being Index [39] and the Hypoglycaemia Fear Survey [40]; (6) several psychological constructs, e.g., self-efficacy [41] and time and risk preferences [33]. In general, standardized questionnaire scales were prioritized; however, new items had to be constructed on topics for which standardized scales were not available, e.g., use of specific insulin pump functions. These items were inspired by measures from the T1D Exchange study [3]. The newly constructed items were discussed and revised several times after tests with insulin pump educators and insulin pump users. The full Steno Tech questionnaire is available as electronic supplementary Table S1. The questionnaire was expected to take approximately $30 \mathrm{~min}$ to complete, and branching logics in REDCap ensured that participants were only asked questions relevant to their diabetes device profile. For example, only respondents using isCGM were asked about scanning frequency. 
Table 1 Demographic and socioeconomic characteristics overall and by response status

\begin{tabular}{|c|c|c|c|c|c|}
\hline Variables & $\begin{array}{l}\text { Study population } \\
(n=1591)\end{array}$ & $\begin{array}{l}\text { Responders } \\
(n=770)\end{array}$ & $\begin{array}{l}\text { Non- } \\
\text { responders } \\
(n=821)\end{array}$ & $\begin{array}{l}P \text { value for } \\
\text { difference* }\end{array}$ & $\begin{array}{l}\text { Missing (responders } \\
\text { non-responders) }\end{array}$ \\
\hline Age, years & $42(28-56)$ & $49(36-60)$ & $33(25-49)$ & $<0.001$ & $1 \mid 12$ \\
\hline Age groups & & & & $<0.001$ & \\
\hline $18-25$ & $277(18)$ & $70(9)$ & $207(26)$ & & $1 \mid 12$ \\
\hline $26-49$ & $734(46)$ & $320(42)$ & $414(51)$ & & \\
\hline $50+$ & $568(36)$ & $379(49)$ & $180(23)$ & & \\
\hline Sex, female & $909(58)$ & $459(60)$ & $450(56)$ & 0.097 & $1 \mid 11$ \\
\hline Marital status, married & $715(45)$ & $439(57)$ & $276(34)$ & $<0.001$ & $1 \mid 11$ \\
\hline Cohabitation & & & & 0.011 & $1 \mid 11$ \\
\hline Lives alone & $287(18)$ & $135(18)$ & $152(19)$ & & \\
\hline $\begin{array}{l}\text { Lives with at least one } \\
\text { other adult }\end{array}$ & $1192(76)$ & $599(78)$ & $593(73)$ & & \\
\hline $\begin{array}{l}\text { Lives only with } \\
\text { child(ren) }\end{array}$ & $100(6)$ & $35(4)$ & $65(8)$ & & \\
\hline Education & & & & $<0.001$ & $15 \mid 44$ \\
\hline $\begin{array}{l}\text { Primary }(7 \text { th }-10 \text { th } \\
\text { grade) }\end{array}$ & $166(11)$ & $61(8)$ & $105(13)$ & & \\
\hline $\begin{array}{l}\text { High school or } \\
\text { vocational school }\end{array}$ & $670(44)$ & $288(38)$ & $382(49)$ & & \\
\hline $\begin{array}{l}\text { Short or medium } \\
\text { higher education }\end{array}$ & $378(24)$ & $217(29)$ & $161(21)$ & & \\
\hline Long higher education & $318(21)$ & $189(25)$ & $129(17)$ & & \\
\hline $\begin{array}{l}\text { Employment status, } \\
\text { employed }\end{array}$ & & & & $<0.001$ & $1 \mid 11$ \\
\hline Employed & $980(62)$ & $519(67)$ & $461(57)$ & & \\
\hline Unemployed & $136(9)$ & $52(7)$ & $84(10)$ & & \\
\hline Retired & $173(11)$ & $114(15)$ & $59(7)$ & & \\
\hline Student & $290(18)$ & $84(11)$ & $206(25)$ & & \\
\hline $\begin{array}{l}\text { Yearly disposable } \\
\text { personal income, } \\
\$ 1000\end{array}$ & $49(26-71)$ & $57(34-78)$ & $39(21-63)$ & $<0.001$ & $1 \mid 12$ \\
\hline
\end{tabular}


Table 1 continued

\begin{tabular}{llllll}
\hline Variables & $\begin{array}{l}\text { Study population } \\
(\boldsymbol{n}=\mathbf{1 5 9 1})\end{array}$ & $\begin{array}{l}\text { Responders } \\
(\boldsymbol{n}=770)\end{array}$ & $\begin{array}{l}\text { Non- } \\
\text { responders } \\
(\boldsymbol{n}=\mathbf{8 2 1})\end{array}$ & $\begin{array}{l}\boldsymbol{P} \text { value for } \\
\text { difference* }\end{array}$ & $\begin{array}{l}\text { Missing (responders } \\
\text { non-responders) }\end{array}$ \\
\hline $\begin{array}{l}\text { Yearly disposable family } \\
\text { income, } \$ 1000\end{array}$ & $73(43-109)$ & $80(48-112)$ & $68(39-105)$ & $<0.001$ & $2 \mid 15$ \\
\hline
\end{tabular}

Descriptive statistics for categorical data are given as frequencies (\%, without missing values) and for continuous data as median (p25-p75). The 'Not married' category includes widow/widower, divorced, longest living of two partners and terminated partnership. The "Short- and medium higher education" category includes degrees from business academy and vocational college educations; the "Long higher education" category includes university degrees (bachelor, master and doctorate degrees). The 'Retired' category includes both early (e.g., due to disability) and timely ( $~ 65$ years) retirees. Yearly disposable income was converted from Danish Kroner to US dollars using the exchange rate of 1 June 2018 $\left(\$ 1=\right.$ DKK6.69); negative income was coded as zero. ${ }^{*} P$ values are for bivariate association tests. Figure 4 depicts adjusted multivariate analysis results

Due to the focus in this article on non-response and external validity, findings from the questionnaire, which are only available for responders, are delimited to descriptive elements of insulin pump and CGM characteristics and general health behaviour. General health behaviour was evaluated according to the Danish Health Authority's recommendations for physical activity, alcohol intake and nutrition (electronic supplementary Table S2).

\section{Statistical Methods}

Data are reported as frequencies and shares, medians with 25th and 75th quartiles or means with standard deviations (SD) depending on variable distributions. Bivariate differences in sample characteristics between responders and non-responders were explored with chi-squared tests for categorical variables; nonparametric $K$ sample tests using chi-squared tests and Student's two-sided $t$ test were used for continuous variables depending on normality. To assess whether non-response was random or if nonresponders differed systematically from responders, odds ratios of replying to the questionnaire were explored with multivariate logistic regression analysis using robust standard errors. Apparently differing characteristics in bivariate analyses between responders and non-responders were included as predictors in the logistic regression model. $P$ values $<0.05$ were considered statistically significant in all analyses. Analyses were performed in Stata 16.

\section{RESULTS}

In the full cohort of 1591 insulin pump users, median age was 42 years, $58 \%$ were women, and $45 \%$ and $76 \%$ were married or lived with at least one other adult, respectively (Table 1 ).

Median HbA1c in the cohort was $58 \mathrm{mmol} / \mathrm{mol}$ (7.5\%); 30\% had an HbA1c < $53 \mathrm{mmol} / \mathrm{mol}$ (7\%). Furthermore, $44 \%$ had a diabetes duration $\geq 25$ years, $10 \%$ had at least one microvascular complication, and $20 \%$ had prevalent cardiovascular disease (Table 2). Within the past 5 years, $7 \%$ had been admitted to a hospital with a diabetic ketoacidosis or severe hypoglycaemia.

\section{Responder Characteristics}

In total, $770(48.4 \%)$ responded to the survey either in full (87\%) or partially (13\%) (Fig. 1). Among responders, 586 (77\%) used an insulin pump with tubing and 175 (23\%) used a patch pump. Most responders used their insulin pump in conjunction with a CGM (77\%) (Table 3). Of these CGM users, more than half had an automated system with insulin suspend function or a hybrid closed-loop system. On average, responders had initiated insulin pump and 
Table 2 Clinical characteristics

\begin{tabular}{|c|c|c|c|c|c|}
\hline Variables & $\begin{array}{l}\text { Study } \\
\text { population } \\
(n=1591)\end{array}$ & $\begin{array}{l}\text { Responders } \\
(n=770)\end{array}$ & $\begin{array}{l}\text { Non- } \\
\text { responders } \\
(n=821)\end{array}$ & $\begin{array}{l}{ }^{*} \boldsymbol{P} \text { value for } \\
\text { difference }\end{array}$ & $\begin{array}{l}\text { Missing (responders } \\
\text { non-responders) }\end{array}$ \\
\hline $\mathrm{HbAlc}, \mathrm{mmol} / \mathrm{mol}$ & $58(51-65)$ & $56(50-62)$ & $60(53-69)$ & $<0.001$ & $1 \mid 8$ \\
\hline HbAlc, \% & $7.5(6.8-8.1)$ & $7.3(6.7-7.8)$ & $7.6(7.0-8.5)$ & $<0.001$ & $1 \mid 8$ \\
\hline HbAlc groups & & & & $<0.001$ & $1 \mid 8$ \\
\hline$<53 \mathrm{mmol} / \mathrm{mol}(<7.0 \%)$ & $467(30)$ & $264(34)$ & $203(25)$ & & \\
\hline $\begin{array}{l}53-<58 \mathrm{mmol} / \mathrm{mol} \\
(7.0-<7.5 \%)\end{array}$ & $297(19)$ & $167(22)$ & $130(16)$ & & \\
\hline $\begin{array}{l}58-<64 \mathrm{mmol} / \mathrm{mol} \\
(7.5-<8.0 \%)\end{array}$ & $352(22)$ & $177(23)$ & $175(22)$ & & \\
\hline $\begin{array}{l}64-<75 \mathrm{mmol} / \mathrm{mol} \\
(8.0-<9.0 \%)\end{array}$ & $322(20)$ & $134(17)$ & $188(23)$ & & \\
\hline$\geq 75 \mathrm{mmol} / \mathrm{mol}(\geq 9 \%)$ & $145(9)$ & $27(4)$ & $118(15)$ & & \\
\hline Cholesterol (total), mmol/l & $4.4(3.9-4.9)$ & $4.4(3.9-5)$ & $4.4(3.9-4.9)$ & 0.396 & $43 \mid 56$ \\
\hline $\mathrm{HDL}, \mathrm{mmol} / \mathrm{l}$ & $1.7(1.4-2.0)$ & $1.7(1.4-2.2)$ & $1.6(1.4-1.9)$ & $<0.001$ & $43 \mid 58$ \\
\hline $\mathrm{LDL}, \mathrm{mmol} / \mathrm{l}$ & $2.2(1.8-2.7)$ & $2.2(1.7-2.7)$ & $2.2(1.8-2.8)$ & 0.551 & $43 \mid 58$ \\
\hline VLDL, $\mathrm{mmol} / \mathrm{l}$ & $0.4(0.3-0.6)$ & $0.4(0.3-0.5)$ & $0.4(0.3-0.6)$ & 0.004 & $60 \mid 81$ \\
\hline Triglycerides, mmol/l & $0.8(0.7-1.2)$ & $0.8(0.6-1.2)$ & $0.9(0.7-1.3)$ & 0.001 & $44 \mid 58$ \\
\hline UACR, mg/g & $6(4-13)$ & $6(4-13)$ & $6(4-13)$ & 0.973 & $34 \mid 70$ \\
\hline Albuminuria & & & & 0.697 & $34 \mid 70$ \\
\hline $\begin{array}{l}\text { Normoalbuminuria } \\
(<30 \mathrm{mg} / \mathrm{g})\end{array}$ & $1321(89)$ & $654(89)$ & $667(89)$ & & \\
\hline $\begin{array}{l}\text { Microalbuminuria } \\
(30-300 \mathrm{mg} / \mathrm{g})\end{array}$ & $129(9)$ & $66(9)$ & $63(8)$ & & \\
\hline $\begin{array}{l}\text { Macroalbuminuria } \\
(>300 \mathrm{mg} / \mathrm{g})\end{array}$ & $37(2)$ & $16(2)$ & $21(3)$ & & \\
\hline $\begin{array}{l}\text { Thyrotropin } \\
(\mathrm{TSH}), \times 10^{-3} \mathrm{IU} / \mathrm{l}\end{array}$ & $1.7(1.2-2.4)$ & $1.6(1.1-2.4)$ & $1.7(1.2-2.4)$ & 0.892 & $34 \mid 53$ \\
\hline Diabetes duration & & & & $<0.001$ & $0 \mid 0$ \\
\hline $0-24$ years & $893(56)$ & $352(46)$ & $541(66)$ & & \\
\hline$\geq 25$ years & $698(44)$ & $418(54)$ & $280(34)$ & & \\
\hline \multicolumn{6}{|l|}{$\begin{array}{l}\text { Acute diabetes complications, } \\
1 \text { - and } 5 \text {-year history }\end{array}$} \\
\hline Severe hypoglycaemia, 1 year & $29(2)$ & $13(2)$ & $16(2)$ & 0.698 & $0 \mid 0$ \\
\hline
\end{tabular}


Table 2 continued

\begin{tabular}{|c|c|c|c|c|c|}
\hline Variables & $\begin{array}{l}\text { Study } \\
\text { population } \\
(n=1591)\end{array}$ & $\begin{array}{l}\text { Responders } \\
(n=770)\end{array}$ & $\begin{array}{l}\text { Non- } \\
\text { responders } \\
(n=821)\end{array}$ & $\begin{array}{l}{ }^{*} P \text { value for } \\
\text { difference }\end{array}$ & $\begin{array}{l}\text { Missing (responders } \\
\text { non-responders) }\end{array}$ \\
\hline $\begin{array}{l}\text { Severe hypoglycaemia, } \\
5 \text { years }\end{array}$ & $108(7)$ & $46(6)$ & $62(8)$ & 0.211 & $0 \mid 0$ \\
\hline Diabetic ketoacidosis, 1 year & $30(2)$ & $5(1)$ & $25(3)$ & $<0.001$ & $0 \mid 0$ \\
\hline $\begin{array}{l}\text { Diabetic ketoacidosis, } \\
5 \text { years }\end{array}$ & $108(7)$ & $28(4)$ & $80(10)$ & $<0.001$ & $0 \mid 0$ \\
\hline \multicolumn{6}{|l|}{ Microvascular complications } \\
\hline Retinopathy (any) & $155(10)$ & $84(11)$ & $71(9)$ & 0.129 & $0 \mid 0$ \\
\hline Neuropathy & $266(17)$ & $146(19)$ & $120(15)$ & 0.020 & $0 \mid 0$ \\
\hline Nephropathy & $173(11)$ & $91(12)$ & $82(10)$ & 0.241 & $0 \mid 0$ \\
\hline \multicolumn{6}{|l|}{$\begin{array}{l}\text { Macrovascular/other } \\
\text { complications 5-year } \\
\text { history }\end{array}$} \\
\hline Cardiovascular disease & $316(20)$ & $190(25)$ & $126(15)$ & $<0.001$ & $0 \mid 0$ \\
\hline Cerebrovascular disease & $39(2)$ & $17(2)$ & $22(3)$ & 0.548 & $0 \mid 0$ \\
\hline Atherosclerosis & $20(1)$ & $12(2)$ & $8(1)$ & 0.296 & $0 \mid 0$ \\
\hline Chronic kidney disease & $24(2)$ & $12(2)$ & $12(1)$ & 0.874 & $0 \mid 0$ \\
\hline Diabetic foot ulcer & $26(2)$ & $16(2)$ & $10(1)$ & 0.176 & $0 \mid 0$ \\
\hline Amputation of feet or legs & $11(1)$ & $8(1)$ & $3(0)$ & 0.105 & $0 \mid 0$ \\
\hline $\begin{array}{l}\text { Charlson comorbidity index, } \\
\text { mean (SD) }\end{array}$ & $0.7(0.8)$ & $0.8(0.8)$ & $0.6(0.9)$ & 0.001 & $0 \mid 0$ \\
\hline \multicolumn{6}{|l|}{$\begin{array}{l}\text { Psychiatric illness 5-year } \\
\text { history }\end{array}$} \\
\hline Depression & $41(3)$ & $15(2)$ & $26(3)$ & 0.125 & $0 \mid 0$ \\
\hline Anxiety & $15(1)$ & $5(1)$ & $10(1)$ & 0.241 & $0 \mid 0$ \\
\hline
\end{tabular}

Descriptive statistics for categorical data are given as frequencies (\%, without missing values) and for continuous data as medians (25th-75th percentiles). Cardiovascular disease includes (1) ischaemic heart disease, (2) heart failure, (3) hypertensive disease and (4) atrial fibrillation/flutter. Cerebrovascular disease includes (1) stroke (ischaemic and haemorrhagic) and (2) transient ischaemic attack. Chronic kidney disease (CKD) includes (1) moderate CKD, (2) severe CKD and (3) end-stage CKD (including dialysis and kidney transplant). ${ }^{*} P$ values are for bivariate association tests. Figure 4 depicts adjusted multivariate analysis results 
Table 3 Device characteristics of responders $(n=770)$

\begin{tabular}{|c|c|c|}
\hline Variables & $\begin{array}{l}\text { Descriptive } \\
\text { statistics }(n=770)\end{array}$ & Missing \\
\hline $\begin{array}{l}\text { Insulin pump duration, } \\
\text { years }\end{array}$ & $9(6-13)$ & 11 \\
\hline $\begin{array}{l}\text { Age at insulin pump } \\
\text { start, years }\end{array}$ & $39(27-49)$ & 12 \\
\hline Insulin pump type & & 9 \\
\hline $\begin{array}{l}\text { Insulin pump with } \\
\text { tubing }\end{array}$ & $586(77)$ & \\
\hline Insulin patch pump & $175(23)$ & \\
\hline Glucose monitor type & & 23 \\
\hline $\mathrm{BGM}^{\mathrm{a}}$ & $170(23)$ & \\
\hline is $\mathrm{CGM}^{\mathrm{b}}$ & $204(27)$ & \\
\hline $\mathrm{CGM}^{\mathrm{c}}$ & $373(50)$ & \\
\hline Sensor duration, years & $4(2-7)$ & \\
\hline Age at sensor start, years & $45(31-55)$ & 1 \\
\hline Insulin delivery system & & 23 \\
\hline Insulin pump + BGM & $170(23)$ & \\
\hline $\begin{array}{l}\text { Insulin } \\
\text { pump + CGM/ } \\
\text { isCGM/SAP }\end{array}$ & $268(36)$ & \\
\hline $\begin{array}{l}\text { Insulin pump }+\mathrm{LGS}^{\mathrm{e}} / \\
\mathrm{PLGS}^{\mathrm{f}}\end{array}$ & $247(33)$ & \\
\hline $\begin{array}{l}\text { Insulin } \\
\text { pump }+\mathrm{HCL}^{\mathrm{g}} / \mathrm{DIY} \mathrm{Y}^{\mathrm{h}}\end{array}$ & $62(8)$ & \\
\hline
\end{tabular}

Descriptive statistics for categorical data are given as frequencies (\%, without missing values) and for continuous data as median (p25-p75) or mean (standard deviation)

${ }^{a}$ Blood glucose monitor

${ }^{b}$ Intermittently scanned continuous glucose monitor

${ }^{\mathrm{c} C o n t i n u o u s ~ g l u c o s e}$ monitor

${ }^{\mathrm{d}}$ Sensor-augmented insulin pump

${ }^{\mathrm{e}}$ Low glucose suspend

${ }^{\text {f}}$ Predictive low-glucose suspend

${ }^{\mathrm{g}}$ Hybrid closed loop

${ }^{\mathrm{h}}$ Do-it-yourself closed-loop systems sensor treatment, respectively, approximately 9 and 4 years prior to answering the questionnaire. Questions about general health behaviour revealed that $34 \%$ of responders met the Danish Health Authority's recommendations for physical activity level and 38\% for nutrition, $84 \%$ of men and $85 \%$ of women drank within the recommended maximum interval of alcohol units, and that $12 \%$ were smokers (electronic supplementary Table S2). Information on hypoglycaemia awareness was available in $97 \%$ of responders' EMRs and showed that 59\% had normal awareness, 21\% had impaired awareness, and $17 \%$ had complete unawareness. Median BMI in the responder group was 26.5; median blood pressure was 129/77 $\mathrm{mmHg}$.

\section{Responders Versus Non-Responders}

Distributions of (non)response rates by age groups indicated that relatively fewer 18-25 and 26-49 year olds answered the survey (Fig. 2). Bivariate analyses of differences between responders and non-responders showed that responders were older (49 years versus 33 years, $p<0.001$ ) (Table 1). Responders also had significantly lower HbA1c compared with non-responders $\quad(\Delta=-4 \mathrm{mmol} / \mathrm{mol} \quad[-0.4 \%]$, $p<0.001)$, which was also true within the 26-49 years $(\Delta=-5 \mathrm{mmol} / \mathrm{mol} \quad[-0.5 \%]$, $p<0.001) \quad$ and $50+(\Delta=-3 \mathrm{mmol} / \mathrm{mol}$ [-0.3\%], $p=0.006$ ) age groups (Fig. 3). Other variables were also significantly different between responders and non-responders in bivariate analyses, among others marital status and 5-year history of ketoacidosis events (Tables 1 and 2).

The adjusted multivariate logistic regression analysis showed that 50 + years of age (odds ratio $[\mathrm{OR}]=2.3,95 \%$ confidence interval $[\mathrm{CI}]$ : 1.4-3.8), female sex $(\mathrm{OR}=1.3$, CI: 1.02-1.6), being married $(\mathrm{OR}=1.8, \mathrm{CI}: 1.3-2.4)$ and having long higher education $(\mathrm{OR}=1.6, \mathrm{CI}$ : 1.004-2.5) were significantly associated with a higher likelihood of responding to the survey. Conversely, HbA1c from 64 to $<75 \mathrm{mmol}$ $(8.0-9.0 \%) \quad(\mathrm{OR}=0.6, \quad \mathrm{CI}: \quad 0.4-0.8)$, $\mathrm{HbA} 1 \mathrm{c} \geq 75 \mathrm{mmol} / \mathrm{mol}(\geq 9.0 \%)(\mathrm{OR}=0.2, \mathrm{CI}$ : $0.1-0.3)$ and prevalent ketoacidosis within the 


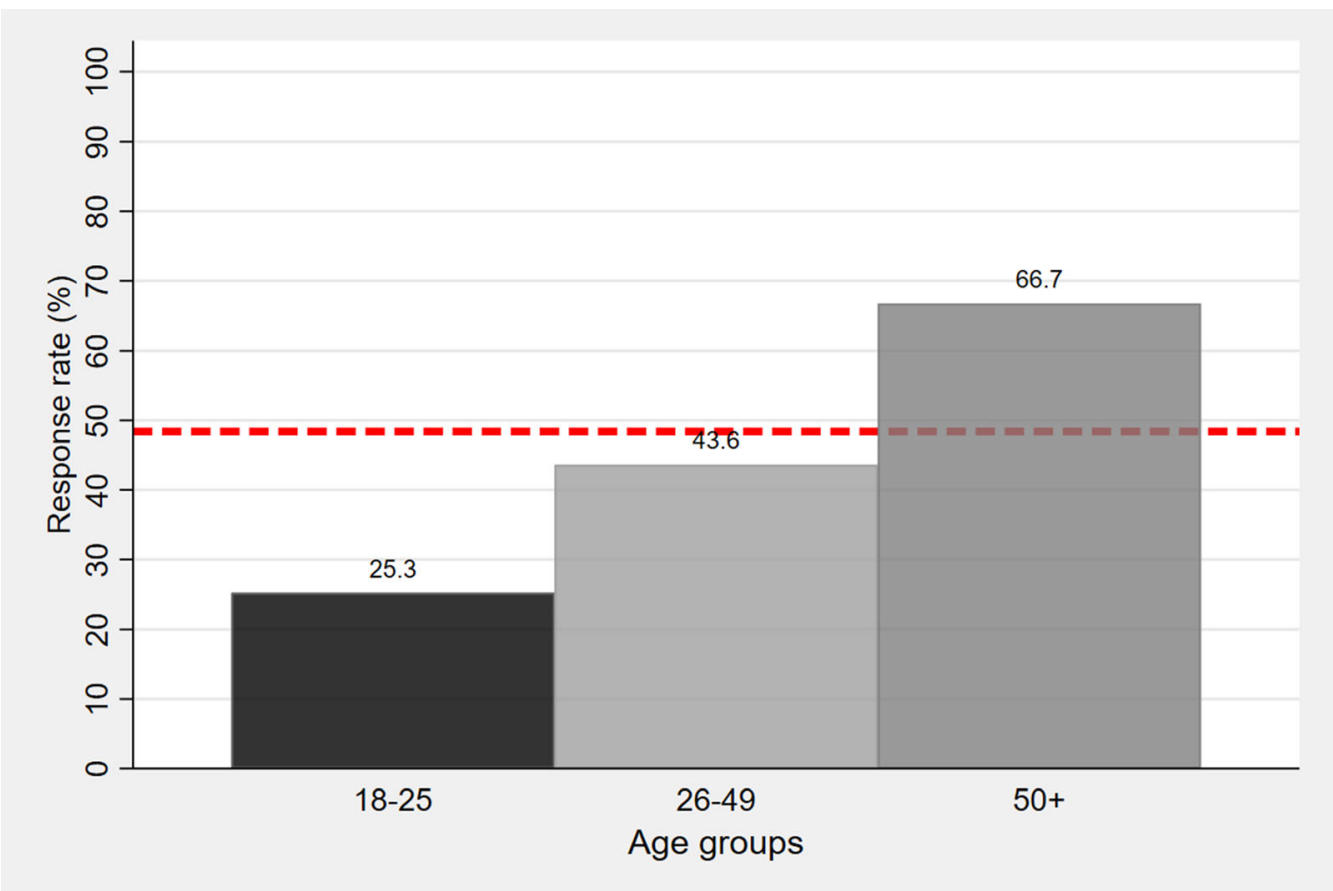

Fig. 2 Response rate distribution by age group. Dashed line indicates the overall response rate (48.4\%)

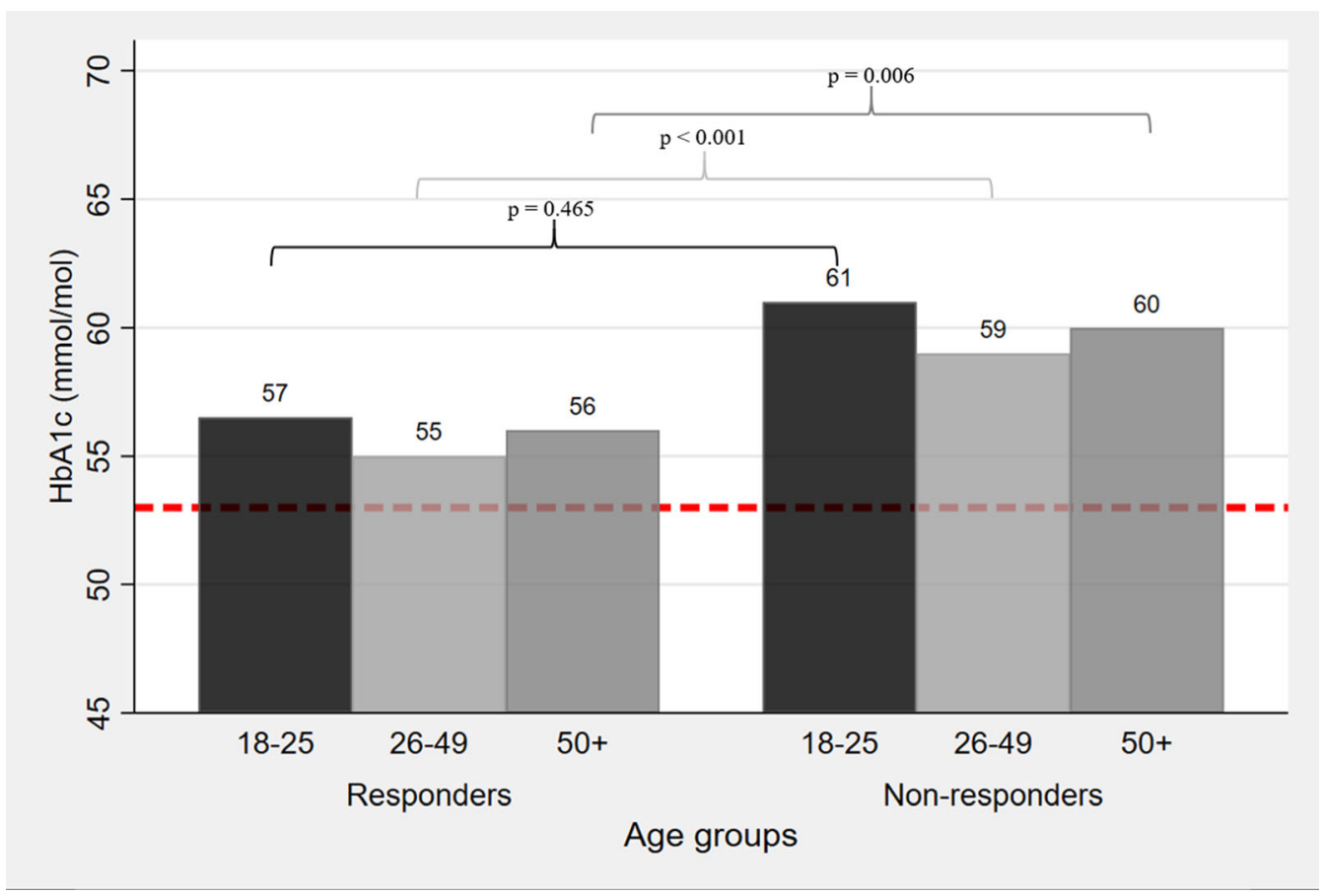

Fig. $3 \mathrm{HbAlc}$ distribution by age group of responders versus non-responders. Dashed line indicates recommended target HbAlc level $(53 \mathrm{mmol} / \mathrm{mol} ; 7.0 \%)$ 


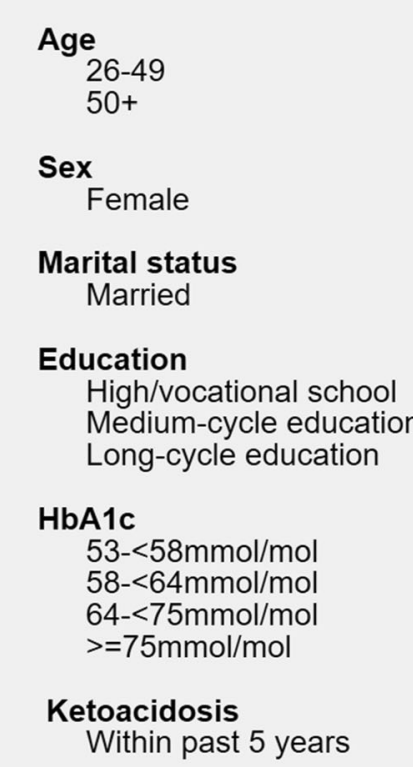

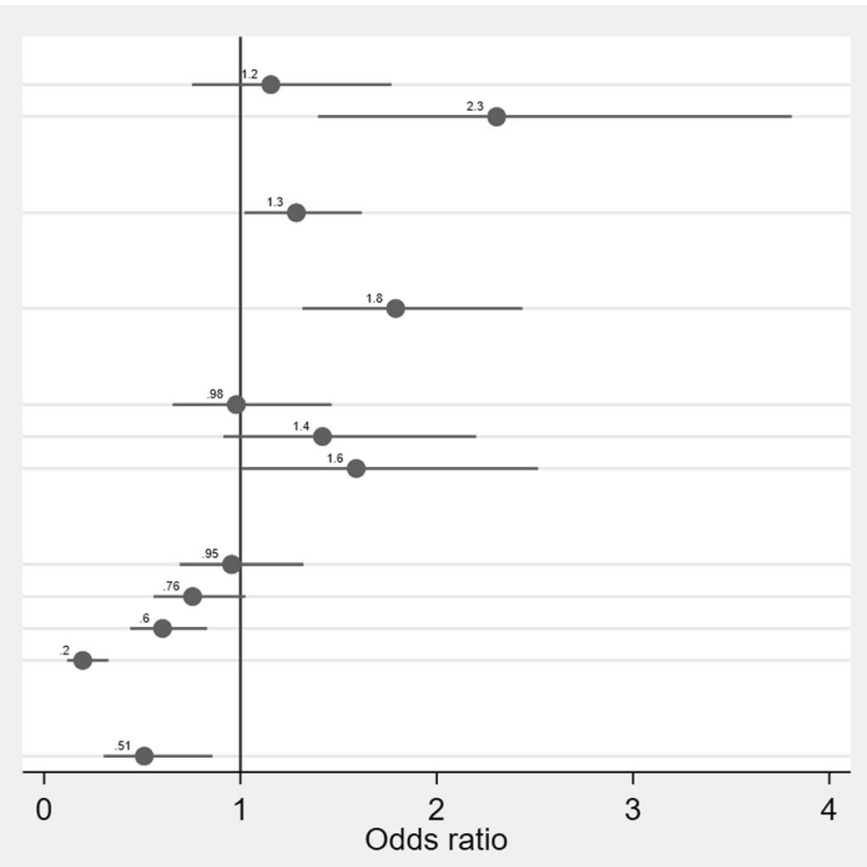

Fig. 4 Odds ratios of responding to the questionnaire. Reference categories: age $=18-25$ years; sex = male; marital status = unmarried; $\quad$ education $=$ primary school; $\mathrm{HbAlc}=<53 \mathrm{mmol} / \mathrm{mol} ; \quad$ ketoacidosis $=$ no. Only variables containing statistically significant associations

past 5 years $(\mathrm{OR}=0.5, \mathrm{CI}: 0.3-0.9)$ were associated with a lower likelihood of responding to the survey (Fig. 4).

\section{DISCUSSION}

In this article, we have described key methodological elements of the Steno Tech Survey study, presented characteristics of the cohort and conducted a thorough analysis of differences between questionnaire-responders and non-responders. In total, 1591 adult insulin pump users with T1D were invited to participate in a questionnaire that assessed aspects of insulin pump self-management and psychosocial health; $48 \%$ responded. Data on demographic, socioeconomic and clinical characteristics for responders as well as non-responders were drawn from national registries. Non-response analysis revealed both similarities are shown. Shown odds ratios are adjusted for one another in the same model together with cohabitation and employment status, income, diabetes duration, neuropathy, cardiovascular disease and Charlson comorbidity index

and disparities between responders and nonresponders.

The most pronounced difference between responders and non-responders concerned age, whereas the youngest participants (18-25 years) were underrepresented among responders (25\%); the opposite was the case with the $50+$ age group for whom the response rate was high (67\%). In the adjusted analysis, younger age was identified as a significant predictor of non-response compared to the oldest age group (50 + years). Furthermore, in the adjusted analysis, female sex, marriage and long higher education were associated with a higher likelihood of responding to the questionnaire; the opposite was the case for HbA1c levels > $64 \mathrm{mmol} / \mathrm{mol}(8.0 \%)$ and prevalent ketoacidosis within the past 5 years. Whereas marriage and longer education were also found to be a significant predictor of responding to questionnaires in other contexts, response rates between women and men differ across contexts $[42,43]$. 
Other socioeconomic characteristics, diabetes complications and comorbidities were not associated with questionnaire response status in the adjusted analysis, so responders may be considered a representative sample of the entire population of insulin pump users in the Capital Region of Denmark in these respects. However, the fact that non-responders on average had higher HbA1c and prevalence of ketoacidosis means that the collected responses may not be completely representative for the entire cohort, especially concerning the young. Thus, in future analyses of the cohort, the missing responses from important sub-groups should be considered carefully.

To limit response bias, the questionnaire was thoroughly tested in interviews and in a pilot test with insulin pump users, and standardized, multi-item questionnaire scales were used to assess psychosocial factors. New items on topics for which standardized questionnaire scales were not available were rigorously tested before use. Due to the many topics covered, the questionnaire was lengthy, in some cases including $>200$ items depending on the responder's device types. In those cases, completion may have taken longer than the estimated $30 \mathrm{~min}$. Studies have shown that the ideal time to complete a survey should be $<20 \mathrm{~min}$ to avoid fatigue, random answering and premature termination, but that these risks can be diminished if the responder considers the survey important and meaningful [44]. However, some respondents might have ended the survey prematurely and others may have opted out entirely for this reason. Another limitation was that the questionnaire was not available to individuals in the target group without access to the internet and/or e-Boks (e.g., on paper); nevertheless, e-Boks coverage was 93\% in the general population of the Capital Region of Denmark at the time of the questionnaire distribution. Fortunately, the use of national registries allowed information on demographics, socioeconomics and biochemical markers to be collected independently of the questionnaire. Accordingly, the use of registries permitted investigation of several characteristics of nonresponders. Notwithstanding the very high validity of data in Danish registries $[26,29,30]$, data on prevalent diagnoses are only available if treated in the secondary care (hospital) sector. Thus, conditions that are typically managed in primary care such as mild depression and anxiety are likely underestimated in this study.

On average, the full study population had a slightly higher HbA1c than the recommended target of $53 \mathrm{mmol} / \mathrm{mol}(7.0 \%)$. This was most prominent among non-responders. HbA1c was, however, also higher than clinical recommendations among responders. Regardless of response status and device usage, the recommended HbA1c was achieved by $30 \%$ in the full cohort. Direct comparison with other cohorts of insulin pump users collected at different points in time is inherently difficult because of constant technological advances as well as sociocultural differences and variances in uptake in different populations. A study of all insulin pump users in the Central Region of Denmark in 2014-2015 found HbA1c, lipids and share of albuminuria cases similar to those found in the present survey [5]. In contrast, in the 2016-2018 US T1D Exchange registry consisting of $63 \%$ insulin pump users, only $21 \%$ of adults achieved target HbA1c [3].

Moving forward, we will explore relationships between questionnaire-assessed practical and psychosocial aspects of insulin pump treatment and register-based outcomes. Among other topics, we will investigate reported unmet needs in education and support regarding insulin pump use, the role of several psychosocial factors in insulin pump treatment and how psychological constructs such as self-efficacy and time preferences may predict treatment outcomes. It is expected that new knowledge about these aspects of insulin pump treatment may inform the development of improved interventions, such as education and support, that may facilitate improved insulin pump treatment of people with T1D. In doing so, we recognize the potential challenges that lie in conducting contemporaneously relevant research in the rapidly developing diabetes technology context. However, we believe that many psychosocial and behavioural challenges will persist regardless of technological advances. 


\section{CONCLUSIONS}

The Steno Tech Survey study represents a valuable source of knowledge about insulin pumptreated adults with T1D and is, to our knowledge, the first of its kind to combine questionnaire-elicited information on numerous selfmanagement and psychosocial aspects of living with an insulin pump with real-world, registerbased data. Access to high-quality data from national registries allowed for an extensive comparison of responders and non-responders with respect to demographic, socioeconomic and clinical parameters. Responders differed from non-responders regarding age, sex, marital status, education, HbA1c and prevalent ketoacidosis, but were similar concerning a range of other factors, such as comorbidities, diabetes complications and income. Future endeavours to study insulin pump users should include analyses of non-responders and put increased emphasis on including groups that typically opt out such as younger people with T1D and groups facing challenges in meeting glycaemic targets.

\section{ACKNOWLEDGEMENTS}

We acknowledge all survey participants as well as those colleagues and insulin pump users at Steno Diabetes Center Copenhagen and Nordsjællands Hospital Hillerød who assisted us in testing the questionnaire. We also thank SDCC Foodlab, Sabine Schade Jacobsen, Anne Grynnerup Skouboe and Betinna Ewers for valuable help with the healthy eating score and the carbohydrate counting task.

Funding. No specific funding or sponsorship was received for this study or publication of this article. The Rapid Service Fee was funded by the authors. Steno Diabetes Center Copenhagen is a public hospital and research institution under the Capital Region of Denmark, which is partly funded by a grant from the Novo Nordisk Foundation.
Authorship. All named authors meet the International Committee of Medical Journal Editors (ICMJE) criteriafor authorship for this article, take responsibility for the integrity of the work as a whole, and havegiven their approval for this version to be published.

Author Contributions. All authors contributed equally in designing the study and developing the questionnaire. Co-first authors Kristoffer P. Madsen and Karen Rytter drafted the manuscript, acquired the data, performed statistical analyses and interpreted results. Henrik U. Andersen contributed with acquisition and interpretation of data and critically reviewed the article. Bryan Cleal, Eva Hommel, Mette A. Nexø and Timothy Skinner critically reviewed the article; Eva Hommel also assisted in acquiring data. Ulrik Pedersen-Bjergaard coconceived the study idea, contributed to acquisition and interpretation of data and critically reviewed the article. Ingrid Willaing coconceived the study idea and critically reviewed the article. Kirsten Nørgaard co-conceived the study idea, contributed with acquisition and interpretation of data and critically reviewed the article. Signe Schmidt planned the study, acquired, analysed and interpreted the data and critically reviewed and revised the article. All co-authors approved the final version before submission.

Prior Presentation. Parts of this study were presented as a poster at the online 14th International Conference on Advanced Technologies $\&$ Treatments for Diabetes in June 2021.

Disclosures. Kristoffer P. Madsen, Ingrid Willaing and Karen Rytter own shares in Novo Nordisk. Henrik U. Andersen owns shares in Novo Nordisk, has served on advisory boards for Novo Nordisk, Abbott and Astra Zeneca and has received lecture fees from Nordic Infucare. Eva Hommel serves as an adviser to Abbott and Novo Nordisk and owns shares in Novo Nordisk. Bryan Cleal and Mette A. Nexø have nothing to disclose. Ulrik Pedersen-Bjergaard sits on advisory boards for AstraZeneca/Bristol Myers Squibb, Novo Nordisk, Sanofi Aventis and Zealand Pharma, has received speaker's 
honorarium from AstraZeneca/Bristol Myers Squibb, Eli Lilly/Boehringer Ingelheim, Medtronic, Novo Nordisk and Sanofi Aventis and has received research grants from Novo Nordisk. Timothy Skinner sits on advisory boards for Novo Nordisk and Liva Health Care and is PI on an EIT Health project grant in partnership with Roche. Kirsten Nørgaard serves as an adviser to Medtronic, Abbott and Novo Nordisk. She owns shares in Novo Nordisk, has received research grants to her institution from Novo Nordisk, Medtronic, Dexcom, Zealand Pharma and Roche Diabetes Care, and has received lecture fees from Medtronic, Roche Diabetes Care, Rubin Medical, Sanofi, Novo Nordisk, Zealand Pharma and Bayer AG. Signe Schmidt reports Medtronic Diabetes advisory board attendance.

Compliance with Ethics Guidelines. The study was approved by the Danish Data Protection Agency (P-2019-812), exempted from review by the Capital Region of Denmark's Research Ethics Committee (19080899) under the National Danish Research Ethics Committe, and conducted in accordance with the Helsinki Declaration of 1964 and its later amendments. Participants provided digital informed consent to participate in the study.

Data Availability. The datasets generated during and/or analysed during the current study are not publicly available because of data protection regulations. Data are accessible to authorised researchers after application to the Danish Health Data Authority.

Open Access. This article is licensed under a Creative Commons Attribution-NonCommercial 4.0 International License, which permits any non-commercial use, sharing, adaptation, distribution and reproduction in any medium or format, as long as you give appropriate credit to the original author(s) and the source, provide a link to the Creative Commons licence, and indicate if changes were made. The images or other third party material in this article are included in the article's Creative Commons licence, unless indicated otherwise in a credit line to the material. If material is not included in the article's Creative Commons licence and your intended use is not permitted by statutory regulation or exceeds the permitted use, you will need to obtain permission directly from the copyright holder. To view a copy of this licence, visit http://creativecommons.org/licenses/by$\mathrm{nc} / 4.0 /$.

\section{REFERENCES}

1. Pickup JC. Is insulin pump therapy effective in Type 1 diabetes? Diabet Med. 2019;36(3):269-78.

2. Naranjo D, Tanenbaum ML, Iturralde E, Hood KK. Diabetes technology: uptake, outcomes, barriers, and the intersection with distress. J Diabetes Sci Technol. 2016;10(4):852-8.

3. Foster NC, Beck RW, Miller KM, et al. State of type 1 diabetes management and outcomes from the T1D exchange in 2016-2018. Diabetes Technol Ther. 2019;21(2):66-72.

4. Jeyam A, Gibb FW, McKnight JA, et al. Marked improvements in glycaemic outcomes following insulin pump therapy initiation in people with type 1 diabetes: a nationwide observational study in Scotland. Diabetologia. 2021;64(6):1320-31.

5. Kampmann U, Madsen LR, Bjerg L, et al. Prevalence and geographical distribution of insulin pump therapy in the Central Denmark Region and its association with metabolic parameters. Diabetes Res Clin Pract. 2018;141:148-55.

6. Walsh J, Roberts R, Weber D, Faber-Heinemann G, Heinemann L. Insulin pump and CGM usage in the United States and Germany: results of a real-world survey with 985 subjects. J Diabetes Sci Technol. 2015;9(5):1103-10.

7. Beck RW, Riddlesworth TD, Ruedy KJ, et al. Effect of initiating use of an insulin pump in adults with type 1 diabetes using multiple daily insulin injections and continuous glucose monitoring (DIAMOND): a multicentre, randomised controlled trial. Lancet Diabetes Endocrinol. 2017;5:700-8.

8. Jeitler K, Horvath K, Berghold A, et al. Continuous subcutaneous insulin infusion versus multiple daily insulin injections in patients with diabetes mellitus: systematic review and meta-analysis. Diabetologia. 2008;51(6):941-51.

9. Benkhadra K, Alahdab F, Tamhane SU, McCoy RG, Prokop LJ, Murad MH. Continuous subcutaneous insulin infusion versus multiple daily injections in individuals with type 1 diabetes: a systematic 
review and meta-analysis. Endocrine. 2017;55(1): 77-84.

10. Dicembrini I, Cosentino C, Monami M, Mannucci E, Pala L. Effects of real-time continuous glucose monitoring in type 1 diabetes: a meta-analysis of randomized controlled trials. Acta Diabetol. 2021;58(4):401-10.

11. Franklin V. Influences on technology use and efficacy in type 1 diabetes. J Diabetes Sci Technol. 2016;10(3):647-55.

12. Barnard KD, Breton MD. Diabetes technological revolution: winners and losers? J Diabetes Sci Technol. 2018;12(6):1227-30.

13. Regionernes Kliniske Kvalitetsudviklingsprogram [The Regions' Clinical Quality Programme]. Dansk Diabetes Database [Danish Diabetes Databse]: National årsrapport 2018/2019 [National annual report 2018/2019]. 2020.

14. Vedrørende behandling med insulinpumper [On treatment with insulin pumps], 2012.

15. Grunberger G, Abelseth JM, Bailey TS, et al. Consensus Statement by the American Association of Clinical Endocrinologists/American College of Endocrinology insulin pump management task force. Endocr Pract. 2014;20(5):463-89.

16. Pickup JC. The evidence base for diabetes technology: appropriate and inappropriate meta-analysis. J Diabetes Sci Technol. 2013;7(6):1567-74.

17. Jayasekara RS, Munn Z, Lockwood C. Effect of educational components and strategies associated with insulin pump therapy: a systematic review. Int J Evid Based Healthc. 2011;9(4):346-61.

18. Payk M, Robinson T, Davis D, Atchan M. An integrative review of the psychosocial facilitators and challenges of continuous subcutaneous insulin infusion therapy in type 1 diabetes. J Adv Nurs. 2018;74(3):528-38.

19. Rytter K, Schmidt S, Rasmussen LN, Pedersen-Bjergaard U, Norgaard K. Education programmes for persons with type 1 diabetes using an insulin pump: A systematic review. Diabetes Metab Res Rev. 2020;37(5):e3412.

20. Schmidt S, Andersen Nexo M, Norgaard O, et al. Psychosocial factors associated with HbA1c in adults with insulin pump-treated type 1 diabetes: a systematic review. Diabet Med. 2020;37(9): 1454-62.

21. Ritholz MD, Smaldone A, Lee J, Castillo A, Wolpert $\mathrm{H}$, Weinger K. Perceptions of psychosocial factors and the insulin pump. Diabetes Care. 2007;30(3): 549-54.

22. Garmo A, Hornsten A, Leksell J. The pump was a saviour for me. Patients experiences of insulin pump therapy. Diabet Med. 2013;30(6):717-23.

23. Grose DN, O'Brien CL, Castle DJ. Type 1 diabetes and an insulin pump: an iterative review of qualitative literature. Practical Diabetes. 2017;34(8): 281-7.

24. Cleal B, Nexo MA, Schmidt S, Rytter K, Norgaard K, Willaing I. Values and control in type 1 diabetes beyond glycemic outcomes: a qualitative interview study of everyday life with an insulin pump. Chronic Illn. 2021. https://doi.org/10.1177/ 17423953211023962 .

25. Harris PA, Taylor R, Thielke R, Payne J, Gonzalez N, Conde JG. Research electronic data capture (REDCap)-a metadata-driven methodology and workflow process for providing translational research informatics support. J Biomed Inform. 2009;42(2): 377-81.

26. Pedersen CB. The Danish civil registration system. Scand J Public Health. 2011;39(7 Suppl):22-5.

27. Jensen VM, Rasmussen AW. Danish education registers. Scand J Public Health. 2011;39(7 Suppl):91-4.

28. Baadsgaard M, Quitzau J. Danish registers on personal income and transfer payments. Scand J Public Health. 2011;39(7 Suppl):103-5.

29. Arendt JFH, Hansen AT, Ladefoged SA, Sorensen HT, Pedersen L, Adelborg K. Existing data sources in clinical epidemiology: laboratory information system databases in Denmark. Clin Epidemiol. 2020;12:469-75.

30. Schmidt M, Schmidt SA, Sandegaard JL, Ehrenstein V, Pedersen L, Sorensen HT. The Danish National Patient Registry: a review of content, data quality, and research potential. Clin Epidemiol. 2015;7: 449-90.

31. Quan H, Li B, Couris CM, et al. Updating and validating the Charlson comorbidity index and score for risk adjustment in hospital discharge abstracts using data from 6 countries. Am J Epidemiol. $2011 ; 173(6): 676-82$.

32. Pedersen-Bjergaard U, Pramming S, Thorsteinsson B. Recall of severe hypoglycaemia and self-estimated state of awareness in type 1 diabetes. Diabetes Metab Res Rev. 2003;19(3):232-40.

33. Madsen KP, Kjaer T, Skinner T, Willaing I. Time preferences, diabetes self-management behaviours 
and outcomes: a systematic review. Diabet Med. 2019;36(11):1336-48.

34. Willis GB. Cognitive interviewing: a tool for improving questionnaire design. SAGE Publications Inc.; 2005. p. 352.

35. Polonsky WH, Fisher L, Hessler D, Edelman SV. Development of a new measure for assessing insulin delivery device satisfaction in patients with type 1 and type 2 diabetes. Diabetes Technol Ther. 2015;17(11):773-9.

36. Polonsky WH, Fisher L, Hessler D, Edelman SV. Development of a new measure for assessing glucose monitoring device-related treatment satisfaction and quality of life. Diabetes Technol Ther. 2015;17(9):657-63.

37. Danquah IH, Skov SS, Callesen BR, Voss I, Petersen $\mathrm{CB}$, Tolstrup JS. Validering af spørgsmål om fysisk aktivitet og siddetid [Validation of questions about physical activity and sitting time]. Copenhagen: Statens Institut for Folkesundhed [National Institute of Public Health]; 2016.

38. Fisher L, Polonsky WH, Hessler DM, et al. Understanding the sources of diabetes distress in adults with type 1 diabetes. J Diabetes Complications. $2015 ; 29(4): 572-7$.
39. Topp CW, Ostergaard SD, Sondergaard S, Bech P. The WHO-5 Well-Being Index: a systematic review of the literature. Psychother Psychosom. $2015 ; 84(3): 167-76$.

40. Grabman J, Vajda Bailey K, Schmidt K, et al. An empirically derived short form of the Hypoglycaemia Fear Survey II. Diabet Med. 2017;34(4): $500-4$.

41. Schwarzer R, Jerusalem M. Generalized self-efficacy scale. In: Weinman J, Wright S, Jonhston M, editors. Measures in health psychology: a user's portfolio Causal and control beliefs. UK: Windsor, UK: NFER-NELSON; 1995. p. 35-7.

42. Linnenkamp U, Gontscharuk V, Brune M, et al. Using statutory health insurance data to evaluate non-response in a cross-sectional study on depression among patients with diabetes in Germany. Int J Epidemiol. 2020;49(2):629-37.

43. Christensen AI, Lau CJ, Kristensen PL, et al. The Danish National Health Survey: Study design, response rate and respondent characteristics in 2010, 2013 and 2017. Scand J Public Health. 2020. https://doi.org/10.1177/1403494820966534.

44. Revilla M, Ochoa C. Ideal and maximum length for a web survey. Int J Mar Res. 2017;59(5):557-65. 